\title{
Electron temperature estimation using the Pulse Height Analysis System at Wendelstein 7-X stellarator
}

\author{
Natalia Krawczyk ${ }^{* a}$, Monika Kubkowska ${ }^{\mathrm{a}}$, Agata Czarnecka ${ }^{\mathrm{a}}$, Slawomir Jablonski ${ }^{\mathrm{a}}$, Marta Gruca ${ }^{\mathrm{a}}$, \\ Tomasz Fornal $^{\mathrm{a}}$, Leszek Ryćc ${ }^{\mathrm{a}}$, Henning Thomsen ${ }^{\mathrm{b}}$, Golo Fuchert ${ }^{\mathrm{b}}$ and W7-X team ${ }^{\mathrm{b}}$
}

\author{
${ }^{a}$ Institute of Plasma Physics and Laser Microfusion, Hery 23 St. 01-497 Warsaw, Poland \\ ${ }^{b}$ Max-Planck-Institut für Plasmaphysik, Wendelsteinstrasse 1, 17491 Greifswald, Germany
}

\begin{abstract}
This paper presents measurements of the electron temperature in Wendelstein 7-X (W7-X) stellarator plasmas heated by electron-cyclotron-resonance (ECRH) during the first operational phase (OP1.1). The analysis of the slope of the observed X-ray continuum emission (in a semi-logarithmic plot) measured by the use of the Pulse Height Analysis (PHA) system, provides the information about the electron temperature $\left(\mathrm{T}_{\mathrm{e}}\right)$ of the hydrogen plasma. The determination of this fundamental plasma parameter is based on the exponential dependence of the continuum radiation (Bremsstrahlung) on photon energy assuming Maxwellian distribution. In this paper, some experimental results of the estimated electron temperature are presented and compared to the results obtained from the simulations of X-ray spectrum and other diagnostic like the Thomson Scattering (TS) system.
\end{abstract}

Keywords: PHA diagnostic, electron temperature, stellarator, Wendelstein 7-X

\section{Introduction}

Wendelstein 7-X (W7-X) [1-2], a large optimized stellarator with superconducting coil system started its first operational phase (OP1.1) at the end of 2015. For OP1.1, the last closed flux surface (LFCS) was defined by five poloidal uncooled limiters made of graphite. The brief experimental campaign allowed the commissioning of installed diagnostic systems and W7-X components as well as the initial physics studies [3-5].

Electron temperature $\left(T_{e}\right)$ as a one of the key plasma parameters, which indicate fusion performance, was routinely measured during the $\mathrm{W} 7-\mathrm{X}$ experiments. In the OP1.1 limiter configuration electron temperatures $T_{e} \leq 8$ $\mathrm{keV}$ have been achieved. The major tools for the $T_{e}$ determination at W7-X are Thomson scattering (TS) [6] and Electron Cyclotron Emissions (ECE) [7] diagnostics. Both systems, which are capable to provide electron temperature profiles, have confirmed good agreement between their results throughout OP1.1 [8].

Measurements of $T_{e}$ can be also performed by the Pulse Height Analysis (PHA) [9-12] system installed at W7-X, which primary purpose is impurity content estimation [13-14] and additionally characterization of nonthermal features of the plasma spectral emission [15]. The PHA spectra also provide information about lineintegrated $T_{e}$, along the line-of-sight, as opposed to measurements from TS and ECE diagnostics. This additional use of PHA system may be helpful in confirming the results obtained by systems mainly intended to determination of this basic plasma parameter. For the investigation described in this paper, a set of data has been selected to demonstrate the system capability to measure $\mathrm{T}_{\mathrm{e}}$.

\section{Experimental setup of the PHA system}

The PHA system commissioned and tested during OP1.1 [16] was designed to provide spectra in the range from $0.7 \mathrm{keV}$ up to $19 \mathrm{keV}$ (for $1 / \mathrm{e}$ of detector response). It consists of 3 Silicon Drift Detectors (SDD) equipped with individual square pinholes and additional exchangeable $\mathrm{Be}$ filters of various thickness, to reduce the number of photon flux that reach the detectors. The first two detectors with a thickness of $450 \mu \mathrm{m}$ and an active area of $10 \mathrm{~mm}^{2}$ are equipped with an 8 - $\mu \mathrm{m}$ Beryllium window, while the third one is characterized by a very thin polymer window. For the presented analysis, data in the range from $0.9 \mathrm{keV}$ up to $10 \mathrm{keV}$ (due to the MCA (multichannel analyzer) settings) have been considered.

Despite the fact that similar systems are used for the measuring the soft- $\mathrm{X}$ ray radiations on many fusion devices, the PHA system working at W7-X stands out among the rest for several reasons. First of all, this diagnostic is characterised by satisfactory time resolution $(100 \mathrm{~ms})$, as well as the spatial resolution, for pinhole size $300 \times 300 \mu \mathrm{m}^{2}$, equal $2.5 \mathrm{~cm}$. Also because of already mentioned additional filter exchanging system and energy resolution better than $200 \mathrm{eV}$, the PHA system provides an opportunity to observe light impurities (carbon (C) and oxygen $(\mathrm{O})$ ) what may be relevant in terms of monitoring some processes in plasma.

For $<\mathrm{T}_{\mathrm{e}}>$ estimation described in this paper the $1^{\text {st }}$ PHA channel (with additional $25 \mu \mathrm{m}$ of Be filter) signals obtained for the trigger threshold of $1000 \mathrm{eV}$, the peaking time of $1 \mu \mathrm{s}$ and the pinhole size of $300 \times 300 \mu \mathrm{m}^{2}$ were 
employed. This was because, during the OP1.1 only $1^{\text {st }}$ PHA channel has been optimized to minimize pileup effect. In Fig. 1 the line-of-sight (LOS) of detector 1 is shown. The LOS simulation, which is based on flux surface data (VIMEC) from magnetic configuration $\mathrm{J}$ (used during OP1.1)), shows that the mentioned detector should view almost (about 8,5 cm) through the plasma center.

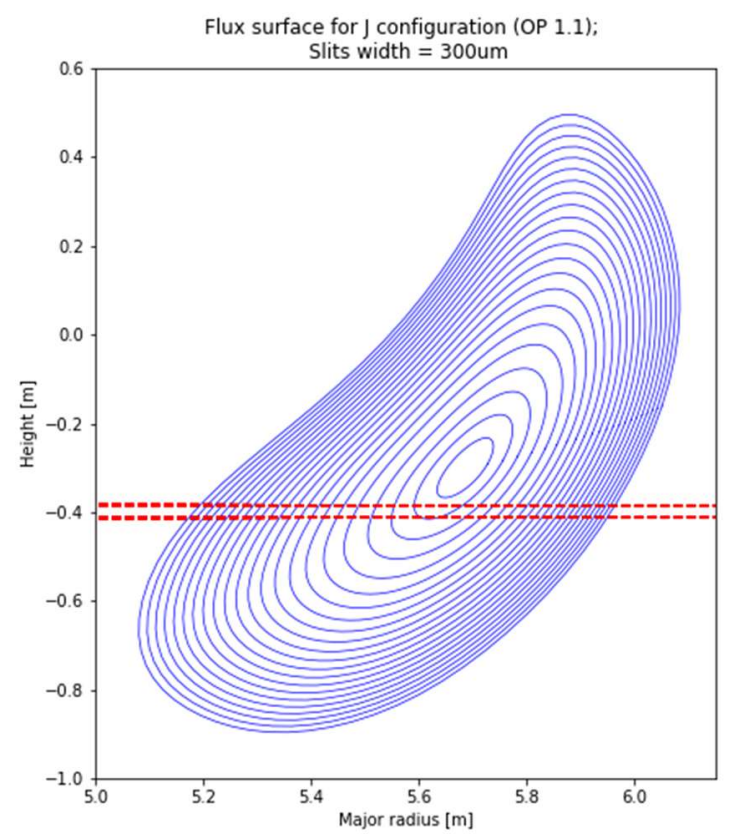

Fig. 1. Flux surfaces (blue) in the poloidal plane with the lineof-sight (red) observed by PHA Channel 1. Flux surfaces taken from VMEC calculation for W7-X program \#20160309.025.

\section{Determination of the electron temperature from the PHA spectra - theoretical description}

The soft X-ray radiation from plasmas, is composed of free-free (Bremsstrahlung), free-bound (recombination radiation) and bound-bound (line radiation) emissions. The first two types of radiation form a continuum spectrum. The equation for hydrogenic bremsstrahlung plasma is as follows [17]:

$\left(\frac{d P_{f f}}{d V d E}\right) f f=3 \cdot 10^{-15} n_{e} \sum_{i} n_{i} Z_{i}^{2} T_{e}^{\frac{-1}{2}} g_{f f} e^{\frac{-E}{k_{B} T_{e}}}$

where $\mathrm{dP}_{\mathrm{ff}}[\mathrm{W}]$ is the power radiated by an impurity ion with the ion charge $Z_{i}$ and ion density $n_{i}$ per unit volume $\mathrm{dV}\left[\mathrm{m}^{3}\right]$ as well as the energy interval $\mathrm{dE}[\mathrm{eV}] . \mathrm{T}_{\mathrm{e}}[\mathrm{eV}], \mathrm{n}_{\mathrm{e}}$ and $\mathrm{E}[\mathrm{eV}]$ are the electron temperature, electron density and photon energy, respectively. $\mathrm{g}_{\mathrm{ff}}$ is the Maxwellaveraged Gaunt factor, which is approximately equal 1 in most cases [Weller].

As in the free-bound case the radiation intensity is given by [15]:

$$
\begin{aligned}
& \left(\frac{d P_{f b}}{d V d E}\right) f b=3 \cdot 10^{-15} n_{e} \sum_{i} n_{i} Z_{i}^{2} T_{e}^{-\frac{1}{2}} g_{f b} e^{-\frac{E}{k_{b} T_{e}}} \beta \\
& \beta=\frac{\xi}{n_{0}^{3}} \frac{\chi_{Z-1}^{n_{0}}}{T_{e}} e^{-\frac{\chi_{Z-1}^{n_{0}}}{k_{b} T_{e}}}+\sum_{n>n_{0}} \frac{2}{n^{3}} \frac{\chi_{Z-1}^{n_{0}}}{T_{e}} e^{-\frac{Z^{2} \chi_{H}}{n^{2} k_{b} T_{e}}}
\end{aligned}
$$

Here, continuum radiation is due to the radiative recombination of ions with charge $\mathrm{Zi}$ to $\mathrm{Zi}-1 . \chi_{\mathrm{H}}$ is the ionization potential of hydrogen, $\chi_{i}$ is the ground state ionization potential and $\mathrm{n}$ is ground state principal quantum number.

The principle of electron temperature determination from the continuum [11-12], is based on the exponential dependence describing bremsstrahlung radiation (eq.1). On the assumption that $g_{f f}$ is independent of energy, $T_{e}$ is directly calculated form the slope $\left(a=-\frac{1}{T_{e}}\right.$ ) of a straight line fitted to the continuum radiation (in a semi-logarithmic plot of $\mathrm{dP}_{\mathrm{ff}} / \mathrm{dVdE}$ vs Energy)

Additionally, the results obtained from experimental spectra are compared with the $\mathrm{T}_{\mathrm{e}}$ from simulations to see if they agreed. In order to simulate the X-ray spectra (the continuum radiation in this particular case), a numerical code RayX was used. It is based on coronal equilibrium of impurities in hydrogen plasma. Moreover, in code, plasma inside cone of view of PHA diagnostic is divided into many subregions. In each subregions it is assumed that $T_{e}$ and $n_{e}$ are constant. In turn, the values of these specific parameters are taken from electron temperature $\mathrm{T}_{\mathrm{e}}(\mathrm{r})$ and density $\mathrm{n}_{\mathrm{e}}(\mathrm{r})$ profiles delivered by Thomson Scattering diagnostic. More detailed description of this code can be found in [18].

\section{Experimental results}

The results of the electron temperature estimation from PHA data presented in this section were obtained from the plasma discharge \#20160309.025. This experiment featured a hydrogen plasma with argon tracer impurities for diagnostic purposes and ECRH heating at a stable level of $2 \mathrm{MW}$. The discharge duration was $700 \mathrm{~ms}$.

(a)

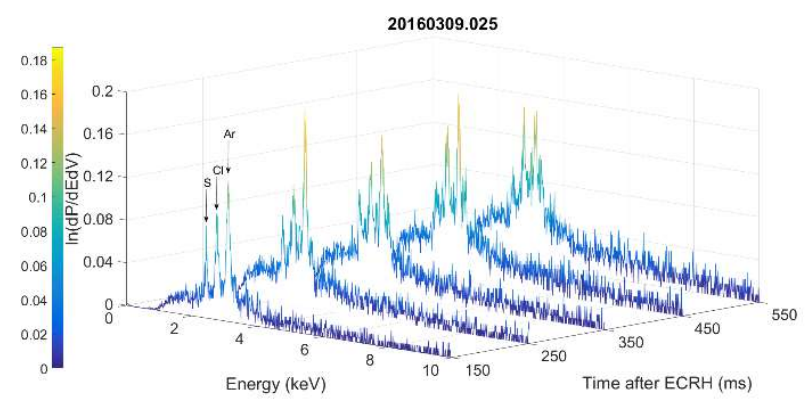

(b) 


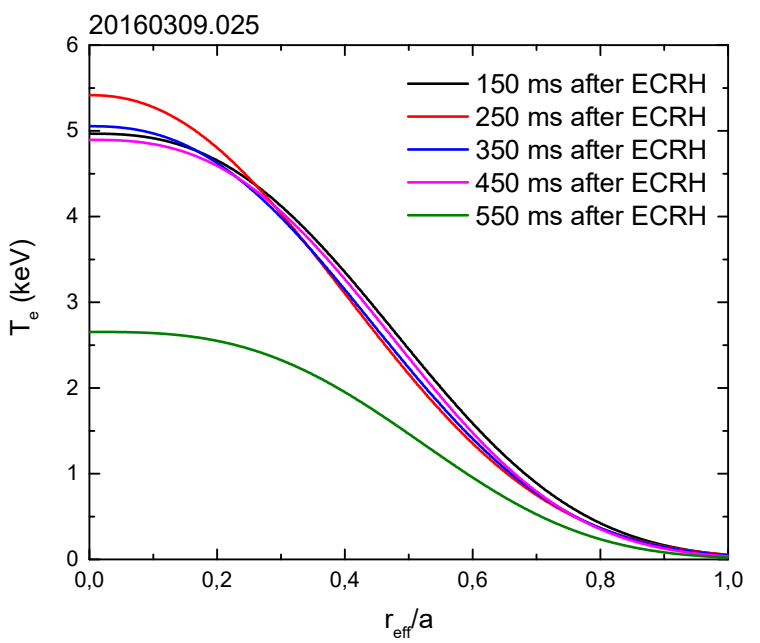

Fig.2. (a) Spectra at particular time frames for W7-X program \#20160309.025 collected by PHA channel 1 and (b) corresponding $\mathrm{Te}(\mathrm{r})$ profiles provided by Thomson Scattering system

For this analysis, five time frames with PHA spectra have been used (cf. Fig 2). Each of them contains data from a $100 \mathrm{~ms}$ time interval. The first frame shows the data recorded $150 \mathrm{~ms}$ after ECRH start, while the last time frame is taken after $550 \mathrm{~ms}$. In Figure 3, the spectra from the selected time frames are presented. In figure $2(\mathrm{~b})$ the corresponding $\mathrm{Te}(\mathrm{r})$ profiles measured by Thomson scattering diagnostic (TS) are shown. The experimental points were fitted with the use of equation:

$T_{e}(r)=T_{e 0}\left[1-\left(\frac{r}{a}\right)^{m}\right]^{n}$

where $\mathrm{T}_{\mathrm{e} 0}$ is the central electron temperature, $\mathrm{r}$ is the effective radius, $\mathrm{a}$ is the minor radius, which was defined by the limiters in OP1.1 to $\sim 0.49 \mathrm{~m}$ (in $\mathrm{r}_{\text {eff }}$ ) and $\mathrm{m}, \mathrm{n}$ are interpolation coefficients.

The choice of these particular time frames was dependent on two major factors. First, the quality of data provided by the PHA system needs to have feasible statistics (above $\sim 10000$ counts, negligible pile-up effects) which allows to observe clear continuum radiation. Second, the profiles of the electron temperature $\mathrm{T}_{\mathrm{e}(\mathrm{r})}$ and density $\mathrm{n}_{\mathrm{e}(\mathrm{r})}$ required for simulation should be satisfactory (physically acceptable). The procedure of estimation the electron temperature $\left\langle\mathrm{T}_{\mathrm{e}}\right\rangle$ is illustrated in Fig. 3 on the example of the frame number 2 (250 ms after ECRH start).

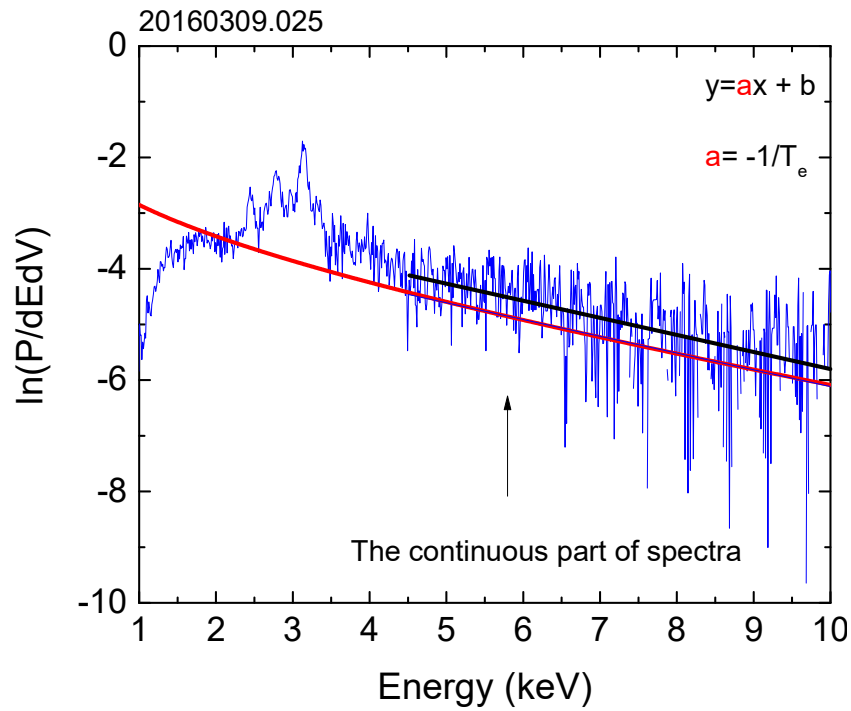

Fig. 3. Determination of the electron temperature from the slope of continuum radiation - on the basis of the PHA spectra(blue) and simulated one from RayX code (red).

In a semi-logarithmic plot of X-ray radiated power emission into energy interval and volume as a function of energy, the PHA spectrum (blue line) and simulated continuum radiation (red line) are presented. For both cases, experimental and simulated data, straight lines were fitted to the bremsstrahlung (using the method of least squares) in the energy range between $4.5 \mathrm{keV}$ and 10 $\mathrm{keV}$. This energy range was chosen because it is free of line radiation. For this specific frame $250 \mathrm{~ms}$ after ECRH start, $\mathrm{T}_{\mathrm{e}(\mathrm{r})}$ and $\mathrm{n}_{\mathrm{e}(\mathrm{r})}$ profiles (from a fit to the TS data, cf. Fig $2 b$ ) used in simulation have a central electron temperature $\mathrm{T}_{\mathrm{e} 0}=5.4 \mathrm{keV}$ and central electron density $\mathrm{n}_{\mathrm{eo}}$ $=1.92 \times 10^{19} 1 / \mathrm{m}^{3}$ (both values are taken from Thomson measurements). As result, the average temperature estimated from the slope of the simulated continuum, is $<\mathrm{T}_{\mathrm{e}, \mathrm{sim}}>=(3.320 \pm 0.004) \mathrm{keV}$, while the result from the experimental PHA spectrum is $\left\langle\mathrm{T}_{\mathrm{e}, \exp }\right\rangle=(3.22 \pm 0.22)$ $\mathrm{keV}$. These values show good agreement between simulation based on TS data and PHA data analysis within the error bars. In the Tab.1, results for all five time frames of the pulse \#20160309.025 are shown. The values of $\left.<\mathrm{T}_{\mathrm{e}}\right\rangle$ estimated from both analyzes are very similar, especially for frames 2 and 3. The biggest differences between the obtained results appear, when the PHA spectra recorded a small number counts (below 10000 counts/time frame) which leads to higher uncertainties in bremsstrahlung (frame 1). Also for frame 5, there is a discrepancy between experimental and simulation data. This is due to the fact that during OP1.1, the PHA time frames were not of equal length $(100 \mathrm{~ms} \pm 50 \mathrm{~ms}$, usually $\sim 105 \mathrm{~ms}$ ), what led to time shifts. Both, in $\left\langle\mathrm{T}_{\mathrm{e}}\right\rangle$ from PHA spectra and simulations based on TS profiles, the errors were calculated as standard deviation of the line fitted to the Bremsstrahlung, due to the fact that the main source of errors in presented discharges is the statistics of the recorded counts. 
Table 1. Comparison of the electron temperature estimated from the PHA spectra and simulations for discharge \#20160309.025

\begin{tabular}{ccc}
\hline $\begin{array}{c}\text { Time after } \\
\text { ECRH start } \\
(\mathrm{ms})\end{array}$ & $\begin{array}{c}<\mathrm{Te}>\text { from } \\
\text { PHA } \\
\text { spectra } \\
(\mathrm{keV})\end{array}$ & $\begin{array}{c}<\mathrm{Te}>\text { from } \\
\text { simulations } \\
(\mathrm{keV}) \text { based on TS } \\
\text { profiles }\end{array}$ \\
\hline 150 (frame1) & $3.75 \pm 0.72$ & $3.180 \pm 0.003$ \\
250 (frame2) & $3.22 \pm 0.22$ & $3.320 \pm 0.004$ \\
350 (frame3) & $3.25 \pm 0.24$ & $3.230 \pm 0.004$ \\
450 (frame4) & $3.53 \pm 0.25$ & $3.210 \pm 0.004$ \\
550 (frame5) & $3.55 \pm 0.22$ & $2.050 \pm 0.002$ \\
& & \\
\hline
\end{tabular}

Although, the PHA system is set to observe across the plasma center, $\left\langle\mathrm{T}_{\mathrm{e}}>\right.$ values determined from experimental data are always lower than the central electron temperatures provided by the TS system $\left(\right.$ see $T_{e}\left(r_{e f f}=0\right)$ in fig.2b). This could be explained by the fact that the that TS collects data directly through the plasma center, while the LOS of the 1st PHA channel system is located around $8.5 \mathrm{~cm}$ below the plasma center (according to VMEC equilibrium reconstructions of the flux surfaces).

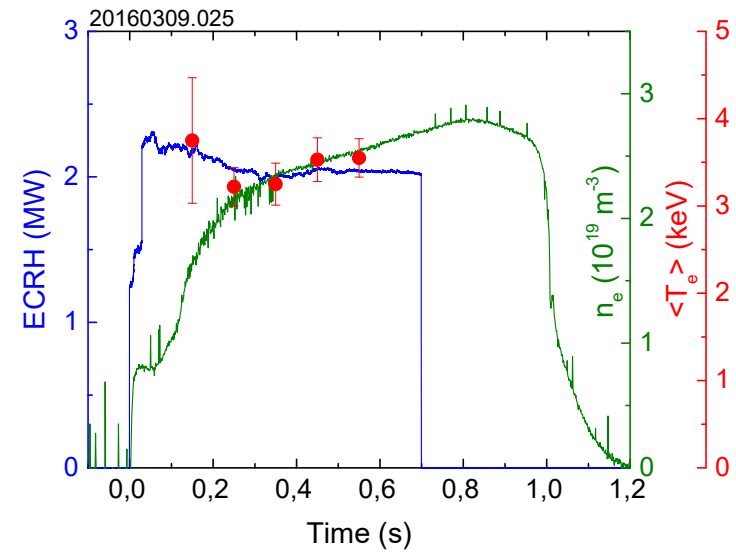

Fig. 4. The average electron temperature $<\mathrm{Te}>$ estimated from the PHA spectra in comparison with ECRH heating and electron density

Moreover, on the basis of the presented pulse, $\left\langle\mathrm{T}_{\mathrm{e}}\right\rangle$ estimated from experimental spectra remains stable during the ECRH heating, which was set at an almost constant level of power ( 2 MW during $700 \mathrm{~ms})$. This typical trend is presented in Fig.4. Additionally, Fig.5 presents experimental results from PHA and TS systems including data from discharge \#20160309.024 with different ECRH steps (from $\sim 0.6 \mathrm{MW}$ to $2 \mathrm{MW}$ ). In this particular case , the maximum difference between $\left\langle\mathrm{T}_{\mathrm{e}}\right\rangle$ (from PHA diagnostic) for the lowest and highest ECRH step is around $1 \mathrm{keV}$, although the heating power was increased by a factor of 4 .

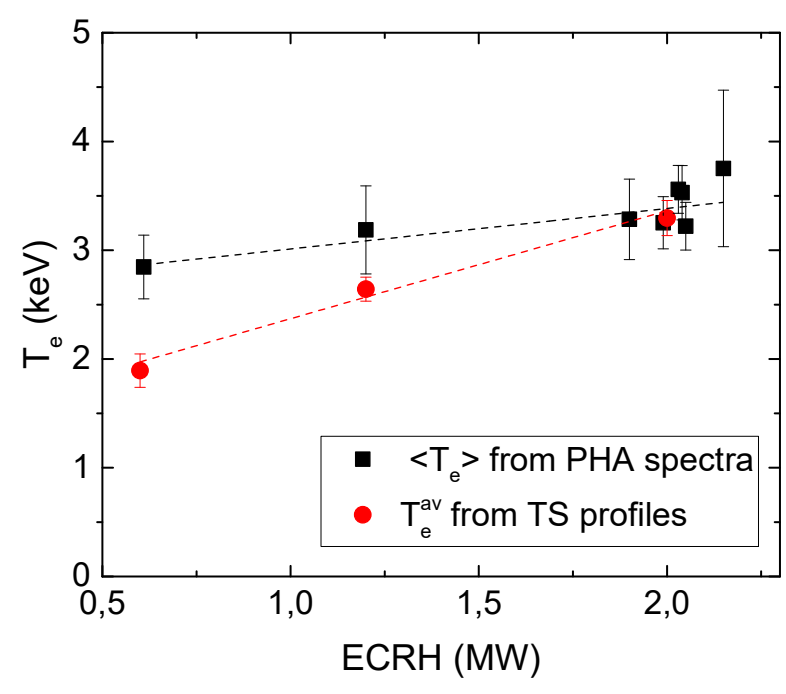

Fig. 5. The change of $\left\langle\mathrm{T}_{\mathrm{e}}\right\rangle$ with ECRH power for experimental data from discharges \#20160309.024 and \#20160309.035

A comparable trend was observed for the data obtained by the TS system. Here, the averaged $T_{e}^{a v}$ was calculated using the following formula:

$T_{a}^{a v}=\frac{\int_{0}^{r} T_{e}(r) n_{e}(r) d r}{\int_{0}^{r} n_{e}(r) d r}$

The maximum difference between ECRH power extremes was $T_{e, \max }^{a v}=1.4 \mathrm{keV}$. The main discrepancy between results obtained by the PHA and TS systems, especially for the lowest value of ECRH heating, results from worst photon statistic collected by PHA system (for this particular case).

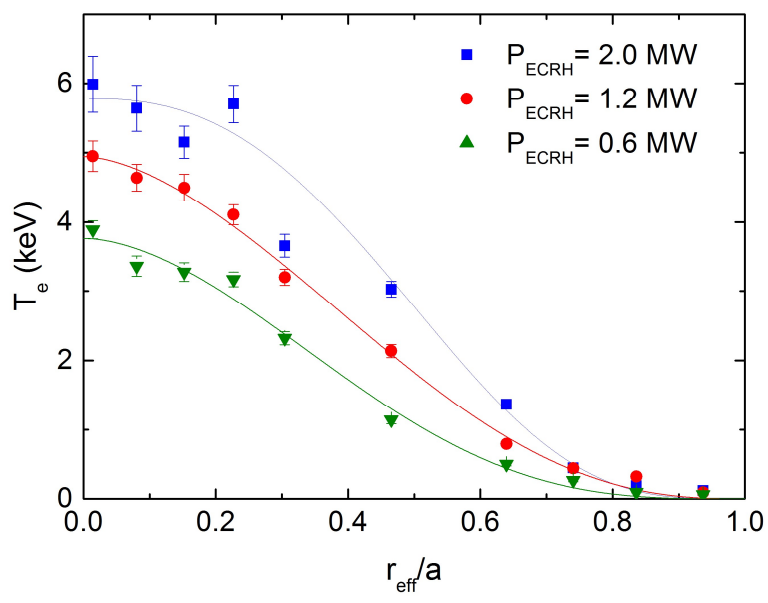

Fig. 6. Te(r) profiles based on measurements provided by Thomson Scattering system from discharge \#20160309.024 with three different ECRH steps.

In Fig.6, The temperature profiles from Thomson scattering for the three ECRH steps are shown.

In order to understand the small responsiveness of the average temperature to the provided heating power, more detailed analysis of the temperature and density profiles are required, which is planned as a next step. 


\section{Conclusions}

First experimental results obtained by the PHA system during the OP1.1, in combination with simulation one, shows that this soft X-ray diagnostic can be a helpful tool for plasma electron temperature determination as well. A correct estimation of $\mathrm{T}_{\mathrm{e}}$ from the continuous part of the spectra was possible in the cases, were collected spectra had sufficient counting statistic. Results obtained by analysis of the experimental data were similar to those, which were derived from the simulated spectra based on Thomson Scattering profiles. Thanks to the PHA acquisition time, which is equal to $100 \mathrm{~ms}$, in most cases it was possible to observe frequent changes of $\mathrm{T}_{e}$ during discharges. However, work on the quality of the data from a single time frame, for further $\mathrm{T}_{\mathrm{e}}$ analysis is needed.

In the next experimental campaign of W7-X (OP1.2), a further optimization of the PHA system is planned. The aim is to measure spectra with higher number of counts and avoiding pile-up effects. As a result, it should be possible to determine $T_{e}$ for all time frames during a typical W7-X pulse.

\section{Acknowledgements}

This work has been carried out within the framework of the EUROfusion Consortium and has received funding from the Euratom research and training programme 20142018 under grant agreement No 633053. The views and opinions expressed herein do not necessarily reflect those of the European Commission.

This scientific work was partly supported by Polish Ministry of Science and Higher Education within the framework of the scientific financial resources in the years 2014-2017 allocated for the realization of the international co-financed project.

\section{References}

[1] H.-S. Bosch et al., Technical challenges in the construction of the steady-state stellarator Wendelstein 7-X Nuclear Fusion 53 (2013) 126001

[2] T. Sunn Pedersen et al., Plans for the first plasma operation of Wendelstein 7-X, Nuclear Fusion 55 (2015) 126001

[3] R. Koenig et al., The Set of Diagnostics for the First Operation Campaign of the Wendelstein 7-X Stellarator, Journal of Instrumentation 10 (2015) P10002

[4] T. Klinger et al., Performence and properties of the first plasmas of the Wendelstein 7-X Stellarator, Plasma Physics and Controloled Fusion (2016) 101221.R1

[5] M. Krychowiak et al., Overview of diagnostic performance and results for the first operation phase in Wendelstein 7-X, Review of Scientific Instruments 87 (2016) 11D304

[6] E. Pasch et al., The Thomson scattering system at Wendelstein 7-X Review of Scientific Instruments 87 (2016) 11E729

[7] S. Schmuck et al., Design of the ECE diagnostic at Wendelstein 7-X, Fusion Engineering and Design 84 (2009)

[8] E. Pasch et al., First results from the Thomson Scattering System at the Stellarator Wendelstein 7-X, 43 ${ }^{\text {rd }}$ EPD
Conference on Plasma Physics

[9] M. Kubkowska et al., Laboratory tests of the Pulse Height Analysis system for Wendelstein 7-X, Journal of Instrumentation 10 (2015) P10016

[10] A. Weller et al., Concepts of X-ray diagnostic for Wendelstein 7-X, Review of Scientific Instruments 75,10 (2014)

[11] A. Weller et al., X-ray Pule Height Analysis on Asdex Upgrade, $38^{\text {th }}$ EPS Conference on Plasma Physics (2011) P5.054

[12] D. Pasini et al., JET X-ray pulse-height analysis system, Review of Scientific Instruments 59 (1988)

[13] M. Kubkowska et al., First Results from the Soft X-ray Pulse Height Analysis System at Wendelstein 7-X Stellarator, 19 ${ }^{\text {th }}$ ISFNT, Kyoto, Japan 2017

[14] A. Czarnecka et al., Study of impurities behaviour in PHA spectra for first magnetic configuration changes in W7-X plasmas, $19^{\text {th }}$ ISFNT, Kyoto, Japan 2017

[15] S. von Goeler et al., Thermal X-ray spectra and impurities in the ST Tokamak, Nuclear Fusion 15 (1975)

[16] N. Krawczyk et al., Commissioning and first operation of the pulse-height analysis diagnostic on Wendelstein 7-X stellarator, Fusion Engineering and Design (2017)

[17] A. Weller et al., Modelling of Soft X-ray Emission from JET Plasmas, JET-IR(87)10

[18] S. Jablonski et al., Simulation of PHA Soft X-Ray Spectra expected from W7-X, Journal of Instrumentation 10 (2015) 